\title{
Performance Evaluation Model of Logistics Enterprises Considering Mass Data in the Internet of Things
}

\author{
Jun $\mathrm{Qi}^{1}$ and Lan $\mathrm{Yi}^{2}$ \\ ${ }^{1,2}$ Department of Accounting, Management School, Jinan University, \\ Guang zhou, 510632, China \\ tqijun@jun.edu.cn or 37883836@qq.com
}

\begin{abstract}
In allusion to such problems as the no use of the structural information of the dataset in the traditional clustering effectiveness evaluation function and the excessive noisy point deletion, the research method integrating theoretical analysis and empirical analysis is adopted to establish KPI management index system model for telecommunication enterprises. A new clustering effectiveness evaluation function is proposed in this article. Specifically, PCA (principal component analysis) method in multivariate statistics is applied in the performance evaluation systems of telecommunication enterprises, and meanwhile relevant instances are analyzed and evaluated. Therein, the evaluation index system has the features of simpleness, strong practicability, low operation cost and high accuracy, and the geometric structure features of dataset are added for the performance evaluation of telecommunication enterprises. Additionally, distance critical value $L$ is added in the compact indexes and the constraint condition thereof is also given in order to construct a new clustering effectiveness evaluation index model which can more scientifically and rationally reflect the actual evaluation result.
\end{abstract}

Keywords:Clustering analysis; Effectiveness evaluation function; Fuzzy soft set; Internet of things; Big data; Comprehensive evaluation; Information integration

\section{Introduction}

The distributed intelligent control based on multi-system (MAS) is not only the future of industrial control, but also another leap during the control science development, wherein the inter-system cooperation is the key control point. In MAS, when one resource finitely encounters the tasks unable to be finished thereby, this resource has to interact and cooperate with other resources in the system so as to form a team to jointly undertake the task, and such team is called as coalition [12]. As a frontier topic of the control theory, coalition issue is always widely concerned by the scholars at home and abroad, and meanwhile rich research achievements have been obtained in the aspects of coalition generation [3-4], coalition formation [5-6], utility allocation [7-8], etc. However, in some complex control and decision-making systems, advantages and disadvantages of coalition utility are directly related to the task completion condition and can be used to judge whether the coalition formed thereby can efficiently and smoothly finish the corresponding tasks. In other words, it is necessary to track and evaluate the working condition of the present coalition in a real-time manner in order to reflect the decision effect and guide the subsequent task execution [9]. Coalition effectiveness is closely related to such factors as member ability, coordination performance, communication cost and member familiarity, and these factors can be only expressed by some fuzzy, summary and uncertain natural linguistic terms 
rather than quantitative numerical values, thus bringing certain difficulty to evaluation [10].

For the evaluation mechanism, the most common evaluation methods include simple weighting method and fuzzy comprehensive evaluation method [11]. These methods usually require the evaluation experts to consider the same evaluation index set in order to give the evaluation information of individuals. In actual evaluation problems, the evaluation experts are usually from different fields, namely different organizations or departments, and each expert has different knowledge and experiences, so each evaluation expert may only concern her/his interesting and familiar indexes in the evaluation index set. If the evaluation experts are required to evaluate all indexes in the evaluation index set, then they will easily get significantly different evaluation results and accordingly cause false judgment, and this is unfavorable for the final decision of the decision-maker. Actually, the fuzzy soft set theory can well handle the above problems.

In allusion to the above problems, an effective coalition evaluation method considering the different individual evaluation index sets of different experts is designed in this article, and meanwhile the fuzzy soft set theory is adopted for the comprehensive evaluation of the coalition. The key point of this method is to adopt the fuzzy soft set theory to handle the individual evaluation index sets of different experts and integrate the evaluation results of different experts on this basis in order to obtain the comprehensive evaluation result of the coalition. The organization structure of the article is as follows: this article firstly describes the coalition evaluation problem, then introduces the fuzzy soft set theory based coalition evaluation process and finally gives experiment results and analysis conclusions.

\section{Big Data Clustering Effectiveness Evaluation Function}

During the effectiveness analysis under noisy environment, in the consideration of the clustering compactness and separability, the noise points shall be included in order to make the effectiveness index sensitive to noise and outliers. MPO clustering effectiveness evaluation function is composed of compactness measurement and separability measurement, wherein the compactness measurement is jointly determined by fuzzy membership matrix and cluster number $c$ and denotes the compactness in the cluster; the separability measurement is defined as the distance among different fuzzy sets and denotes the separation degree of different clusters. On the basis of considering the monotone tendency of PC index along with the increment of cluster number $c$ and improving PX index, $u_{n}$ and $\left(\frac{c+1}{c-1}\right)^{1 / 2}$ $(2 \leq c \leq n)$ are introduced therein as the adjustment index to reduce the influence of the cluster number change on the result, thus to obtain compactness measurement $\operatorname{Com}(U, c)$ :

$\operatorname{Com}(U, c)=\left(\frac{c+1}{c-1}\right)^{1 / 2} \sum_{i=1}^{c} \sum_{j=1}^{n} u^{2} u_{M}$

In the above formula, ${ }_{i j}$ denotes the membership of the $j$ th element belonging to the $i$ th cluster, $u_{M}=\min _{1 \leq \mathrm{i} \leq c} \sum_{j=1}^{n} u^{2}{ }_{i j}$, and $\sum_{j=1}^{n} u_{i j}^{2} / u_{M}$ is used to measure the compactness of cluster ${ }^{i}$ relatively to the most compact cluster. $\operatorname{Com}(U, c)$ denotes 
the compactness of the data in the cluster, and the larger value thereof indicates the better fuzzy division obtained thereby.

In order to obtain correct division in the clustering environment with noise points and outliers, the separability measurement is defined as follows:

$$
\operatorname{Sep}(U, c)=\frac{1}{n} \sum_{j=1}^{n}\left(\sum_{a=1}^{c-1} \sum_{b=a+1}^{c} O_{a b j}(U ; c)\right)
$$

In the above formula, $O_{a b j}(U ; c)=\left\{\begin{array}{l}1-\left|u_{a j}-u_{b j}\right|, i f\left|u_{a j}-u_{b j}\right| \geq T, a \neq b \\ 0, \text { other. }\end{array}, u_{a j}\right.$ and $u_{b j}$ respectively denote the membership values of the $j$ th element to cluster $a$ and $b$.

In $O_{a b j}(U ; c)$, threshold value $\mathrm{T}$ is applied to eliminate the fuzzy data points in the cluster border [14], and these data points are the noise points.

$\operatorname{Sep}(U, c)$ is used to calculate the sum of the separation degrees of all data points in the data set obtained thereby through the membership matrix. The smaller $\operatorname{sep}(U, c)$ value indicates the clearer cluster division.

MPO function is the difference between the compactness measurement and the separability measurement:

$$
M P O(U, V)=\operatorname{Com}(U, c)-\operatorname{Scp}(U, c)
$$

According to the comparison with multiple clustering effectiveness indexes including PC, FS, XB, OS, PACES, CO and W, MPO can well determine the cluster number and can avoid the influence of noise points on data set. However, the structural information of the data set is not used in MPO function, so MPO function is not sensitive to distance. In this article, the geometric structure features of the data set are added on the basis of MPO function in order to avoid the influence of single theory on the detection result. In the separability measurement, the noise points shall be carefully deleted, because excessive deletion will cause data loss. Accordingly, a new critical value $L$ is added in $\operatorname{sep}(c)$ to restrain the noise point scope jointly with the original critical value $T$ in order to avoid result inaccuracy caused by data loss.

\section{Construction of New Clustering Effectiveness Evaluation Function}

\subsection{Compactness Measurement}

In allusion to the problems that MPO function is not directly contacted with the geometric structure of the data set and the clustering effect cannot be comprehensively evaluated, the geometric structure information reflecting the internal compactness in the cluster is integrated in the compactness measurement $\operatorname{Com}(U, c)$ in order to obtain new compactness index $\operatorname{Com}^{\prime}(U, c, d)$.

$$
\operatorname{Com}^{\prime}(U, c, d)=\sum_{i=1}^{c} \frac{\sum_{j=1}^{n} u_{i j}^{2}}{u_{M}{ }^{d}{ }_{M}}\left(\frac{c+1}{c-1}\right)^{1 / 2}
$$


In the above formula, ${ }^{d}{ }_{M}$ is Euclidean distance, and the improved function not only includes the distance from ${ }^{x}$ object to the cluster center ${ }_{v_{i}}$, but also includes the membership function value of ${ }_{j}$ to $\operatorname{cluster} i \cdot \operatorname{Com}^{\prime}(U, c, d)$ value can reflect the compactness of the data points in the cluster, and the larger value indicates the greater compactness of the elements in the cluster and the better division effect.

\subsection{Separability Measurement Based on Dual Constraints to Noise Points}

During the clustering process, noise points and isolated points can significantly influence the clustering result. According to literature [13], the critical value $T$ of the membership is adopted in MPO function to preliminarily separate the noise points under probability condition, but the independent application of probability constraint for defining noise point scope may cause data loss. Therefore, in order to more accurately determine the noise points, the critical value $L$ of the geometric structure is added in this article on the basis of the critical value $T$ of the membership in order to adopt two critical values to jointly determine the noise points and accordingly effectively avoid the data loss under the condition of single theory. The separability measurement combining the probability condition and the geometric structure is defined as follows:

$$
\operatorname{Sep}^{\prime}(U, c, d)=\frac{1}{n \sum_{j=1}^{n} \sqrt[3]{\frac{1}{c} \sum_{i=1}^{c} d_{i j}}} \sum_{j=1}^{n}\left(\sum_{a=1}^{c-1} \sum_{b=a+1}^{c} W(U ; c)\right)
$$

In the above formula,

$$
W(U, c, d)=\left\{\begin{array}{l}
0,\left|u_{a j}-u_{b j}\right| \leq \mathrm{T} \text { and }\left|d_{a j}-d_{b j}\right| \leq L_{j} \text { while } a \neq b . \\
1-\left|u_{a j}-u_{b j}\right| \text { other }
\end{array}\right.
$$

Two critical values $T$ and $L$ are set to eliminate the fuzzy and uncertain points in the cluster border. According to different clustering data distribution structures, values $T$ and $L$ can be given by the experts or customized according to the function. In this article, according to massive data experiments, the two critical values are preliminarily given as follows: $T=0.01$ and $L_{j}=\sqrt[3]{\frac{1}{c} \sum_{i=1}^{c} d_{i j}}$, wherein $d_{i j}$ denotes the distance from the $j$ th element to the $i$ th cluster. $W(U ; c, d)$ is the separation degree of the given data point. The smaller $\operatorname{sep}^{\prime}(c)$ value indicates the clearer cluster division effect.

\subsection{Construction of Clustering Effectiveness Function}

The compactness measurement and the separability measurement are integrated to obtain the following new clustering effectiveness evaluation function:

$$
V S(U, V)=\frac{\operatorname{Com}^{\prime}(U, c, d)}{\operatorname{Sep}^{\prime}(U, c, d)}
$$


A good cluster is required to have $\operatorname{large} \operatorname{Com}^{\prime}(U, c, d)$ and small $\operatorname{Sep}^{\prime}(U, c, d)$, and $V S(U, V)$ is used to define the effectiveness function, wherein the larger $V S(U, V)$ value indicates the better clustering division effect.

\section{Coalition Evaluation Method Based on Fuzzy Soft Set}

\subsection{Coalition Evaluation Process}

As shown in Figure 1 when solving the coalition evaluation problem, firstly, the user shall provide the coalition $R=\left\{r_{1}, r_{2}, \cdots, r_{q}\right\}$ to be evaluated and the evaluation index set $D=\left\{d_{1}, d_{2}, \cdots, d_{n}\right\}$; then, each expert shall give the individual evaluation index set $D_{k}=\left\{d_{1}^{k}, d_{2}^{k}, \cdots, d_{l_{k}}^{k}\right\}$ and the evaluation matrix $V_{k}=\left(v_{t i}^{k}\right)_{q \times l_{k}}$; then, the fuzzy soft set shall be used to integrate the evaluation values obtained from all evaluation experts in order to obtain the final evaluation result.

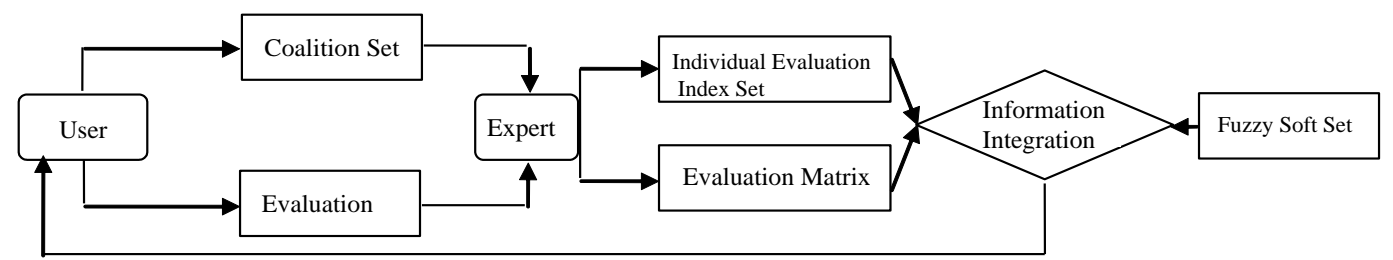

Figure 1. Coalition Evaluation Process Based on Fuzzy Soft Set

4.1.1. Conversion of Evaluation Matrix and Fuzzy Soft Set: According to the individual evaluation set $D_{k}$ and the evaluation matrix $V_{k}$ of each evaluation $\operatorname{expert}^{p_{k}}$, the evaluation information of the evaluation coalition regarding each evaluation index is expressed as the fuzzy soft set ${ }^{\left(F_{k}, D_{k}\right)}$, as shown in formula (8)

$$
\begin{gathered}
\left(F_{k}, D_{k}\right)=\left\{d_{1}^{k}=\left\{r_{1} / v_{11}^{k}, r_{2} / v_{21}^{k}, \cdots, r_{q} / v_{q 1}^{k}\right\},\right. \\
d_{2}^{k}=\left\{r_{1} / v_{12}^{k}, r_{2} / v_{22}^{k}, \cdots, r_{q} / v_{q 2}^{k}\right\}, \\
\vdots \quad \vdots \\
d_{l_{k}}^{k}=\left\{r_{1} / v_{1 l_{k}}^{k}, r_{2} / v_{2 l_{k}}^{k}, \cdots, r_{q} / v_{q l_{k}}^{k}\right\} \\
\}
\end{gathered}
$$

4.1.2. Information Integration: The comprehensive evaluation matrix can be obtained only after the evaluation information of each expert is integrated. The integration method is as follows: implement "AND" operation orderly for the fuzzy soft set ${ }^{\left(F_{1} D_{1}\right)},\left(F_{2}, D_{2}\right), \ldots,{ }^{\left(F_{m}, D_{m}\right)}$. The operation result is expressed by ${ }^{(G, E)}$ as follows:

$(G, E)=\left(G, D_{1} \times D_{2} \times \cdots \times D_{n}\right)=\left(F_{1}, D_{1}\right) \wedge\left(F_{2}, D_{2}\right) \wedge \cdots \wedge\left(F_{m}, D_{m}\right)$

Meanwhile, for $\forall\left(\hat{d}_{1}, \hat{d}_{2}, \cdots, \hat{d}_{n}\right) \in D_{1} \times D_{2} \times \cdots \times D_{m}$, the following formula can be obtained:

$G\left(\hat{d}_{1}, \hat{d}_{2}, \cdots, \hat{d}_{m}\right)=F_{1}\left(d_{1}^{\prime}\right) \cap F_{2}\left(d_{2}^{\prime}\right) \cap \cdots \cap F_{m}\left(d_{m}^{\prime}\right)$ 
Obviously, $(G, E)$ is also a fuzzy soft set. According to definition 2, the parameters of $(G, E)$ are obtained from the combination of the evaluation index sets $D_{1}, D_{2}, \cdots, D_{m}$ of $n$ experts. If $(G, E)$ includes $L$ parameters and $E=\left\{e_{1}, e_{2}, \cdots, e_{L}\right\}$ is true, then $(G, E)$ can be expressed as follows:

$$
\begin{aligned}
& (G, E)=\left\{e_{1}=\left\{r_{1} / \mu_{11}, r_{2} / \mu_{21}, \cdots, r_{q} / \mu_{q 1}\right\},\right. \\
& e_{2}=\left\{r_{1} / \mu_{12}, r_{2} / \mu_{22}, \cdots, r_{q} / \mu_{q 2}\right\}, \\
& \vdots \quad \vdots \\
& e_{L}=\left\{r_{1} / \mu_{1 L}, r_{2} / \mu_{2 L}, \cdots, r_{q} / \mu_{q L}\right\} \\
& \text { \} }
\end{aligned}
$$

In the above formula, $\mu_{t j}$ denotes the coincidence degree between the evaluated coalition $r_{t}$ and the state described by the combined parameter $e_{j}(j=1,2, \cdots, L)$. Two conditions shall be considered for $\mu_{i j}$ :

(1) If $D_{1} \cap D_{2} \cap \cdots \cap D_{m}=\varnothing$ is true, namely: the individual evaluation indexes of the evaluation experts are completely different from each other, then $L=l_{1} \cdot l_{2} \cdots \cdot l_{m}$ is true and any $e_{j}$ can be expressed as $e_{j}=\left(\hat{d}_{1}^{j}, \hat{d}_{2}^{j}, \cdots, \hat{d}_{m}^{j}\right)$, namely: parameter $e_{j}$ is obtained from the combination of parameter $\hat{d}_{1}^{j}$ in $D_{1}$, parameter $\hat{d}_{2}^{j}$ in $D_{2}, \ldots$, parameter $\hat{d}_{m}^{j}$ in $D_{m}$, and $\mu_{t j}$ is determined by formula (12).

$$
\mu_{t j}=\min _{\substack{\hat{d}_{x}^{j} \in\left\{\hat{d}_{1}^{j}, \hat{d}_{2}^{j}, \cdots, \hat{d}_{m}^{j}\right\} \\ k \in\{1,2, \cdots, m\}}}\left\{v_{t x}^{k}\right\}
$$

According to formula (8), if the coordination evaluation made by an expert for a certain coalition is 0.7 and the innovation ability evaluation made by another expert for the same coalition is 0.9 , then the evaluation result obtained after information integration for this coalition is also 0.7, namely: the evaluation value for "good coordination and innovation ability" is 0.7 .

(2) If $D_{1} \cap D_{2} \cap \cdots \cap D_{m} \neq \varnothing$ is true, namely: the individual evaluation indexes of some experts are the same, then $L<l_{1} \cdot l_{2} \cdots \cdot l_{m}$ is true and $e_{j}=\left(\hat{d}_{1}^{j}, \hat{d}_{2}^{j}, \cdots, \hat{d}_{m}^{j}\right)(\hat{m}<m)$, namely: parameter $e_{j}$ is respectively obtained from the combination of $\hat{m}$ different parameters in $D_{1}, D_{2}, \cdots, D_{m}$. If the common evaluation index $d_{c}\left(d_{c} \in D\right)$ exists in $D_{k_{1}}, D_{k_{2}}, \cdots, D_{k_{m}}$, then $\mu_{t j}$ is determined by formula (13).

$$
\begin{aligned}
& \mu_{t j}=\min \left\{\min _{\left\{\begin{array}{l}
\hat{d}_{x}^{j} \in\left\{\hat{d}_{1}^{j}, \hat{d}_{2}^{j}, \cdots, \hat{d}_{m i}^{j}\right\} \\
k \in\left\{1,2, \cdots, \hat{m}^{j}\right\}
\end{array}\right.}\left\{v_{t x}^{k}\right\}, \lambda_{t}\right\} \\
& \lambda_{t}=\underset{k \in\left\{k_{1}, k_{2}, \cdots, k_{m}\right\}}{\operatorname{average}}\left\{v_{t c}^{k}\right\}
\end{aligned}
$$

In other words, when the individual index sets of the evaluation experts have intersection, formula (13) is firstly used to obtain the average value of the same index evaluation values and then formula (14) is used to obtain the average value of the composite index $e_{j}$. 
At present, most researches regarding the fuzzy soft set are only focused on condition (1), namely: the parameter sets are required to be different from each other (without any intersection), but such assumption may be not consistent with the actual situation. However, the method proposed in this article takes the parameter set intersection into consideration.

The evaluation score $\operatorname{Score}\left(r_{t}\right)$ of each coalition to be evaluated is calculated according to $C T$.

$\operatorname{Score}\left(r_{x}\right)=s_{x}-t_{x}$

$s_{x}=\sum_{y=1}^{q} c t_{x y}$

$t_{y}=\sum_{x=1}^{q} c t_{x y}$

In formulae (15) and (16), $s_{x}$ denotes the sum of the $x$ th row in $C T$ and means that the evaluation value of $r_{x}$ is more than the number sum of the evaluation parameters of other members in $R$, and $t_{y}$ denotes the sum of the $y$ th column in $C T$ and means that the evaluation value of $r_{y}$ is more than the number sum of the evaluation parameters of other members in $R$. Therefore, $\operatorname{Score}\left(r_{t}\right)$ denotes the superiority of $r_{t}$ in $R$, namely: the larger $r_{t}$ value indicates the superior $r_{t}$.

Coalition has numerous influencing factors and it is difficult to quantitatively describe these factors by quantitative method, and the significance of using this method to evaluate the coalition lies in the fact that this method can reflect the different preferences of the evaluation experts and the uncertainty of the evaluation information in order to enable the evaluation experts to flexibly express their subjective judgments. Meanwhile, the evaluation information of multiple evaluators can be integrated through "AND" operation of the fuzzy soft set, thus to obtain comprehensive evaluation result.

\section{Instance Analysis}

$R=\left\{r_{1}, r_{2}, r_{3}, r_{4}\right\}$ denotes the coalition to be evaluated, $D=\left\{d_{1}, d_{2}, d_{3}, d_{4}, d_{5}, d_{6}\right\}$ denotes the six attributes of each coalition, namely the evaluation indexes, and $P=\left\{p_{1}, p_{2}, p_{3}\right\}$ denotes three evaluation experts.

(1) Each experts give the individual evaluation index sets $D_{1}=\left\{d_{1}, d_{2}, d_{5}\right\}$, $D_{2}=\left\{d_{1}, d_{3}, d_{4}\right\}$ and $D_{3}=\left\{d_{1}, d_{5}, d_{6}\right\}$ according to the known knowledge and experience and meanwhile give the corresponding evaluation matrixes $V_{1}, V_{2}$ and $V_{3}$.

$$
\begin{aligned}
& \begin{array}{lllllllll}
d_{1} & d_{2} & d_{5} & d_{1} & d_{3} & d_{4} & d_{1} & d_{5} & d_{6}
\end{array}
\end{aligned}
$$

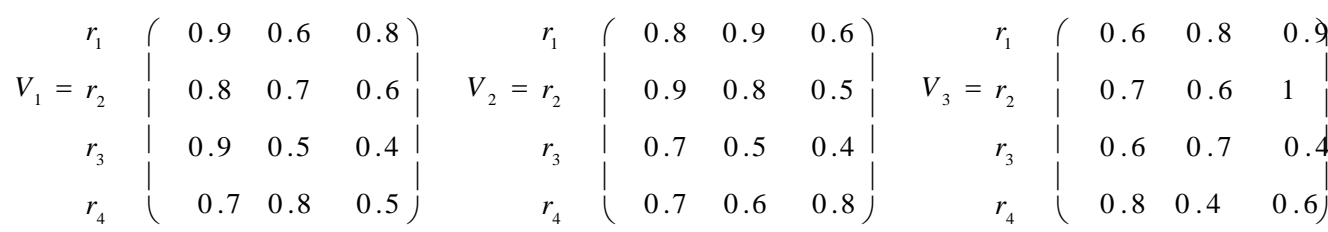

(2) $V_{1}, V_{2}$ and $V_{3}$ are respectively expressed as the fuzzy soft sets $\left(F_{1}, D_{1}\right),\left(F_{2}, D_{2}\right)$ and $\left(F_{3}, D_{3}\right)$. 


$$
\begin{aligned}
\left(F_{1}, D_{1}\right)=\left\{d_{1}\right. & =\left\{r_{1} / 0.9, r_{2} / 0.8, r_{3} / 0.9, r_{4} / 0.7\right\}, \\
d_{2} & =\left\{r_{1} / 0.6, r_{2} / 0.7, r_{3} / 0.5, r_{4} / 0.8\right\}, \\
d_{5} & =\left\{r_{1} / 0.8, r_{2} / 0.6, r_{3} / 0.4, r_{4} / 0.5\right\} \\
\} & \\
\left(F_{2}, D_{2}\right)=\left\{d_{1}\right. & =\left\{r_{1} / 0.8, r_{2} / 0.9, r_{3} / 0.7, r_{4} / 0.7\right\}, \\
d_{3} & =\left\{r_{1} / 0.9, r_{2} / 0.8, r_{3} / 0.5, r_{4} / 0.6\right\}, \\
d_{4} & =\left\{r_{1} / 0.6, r_{2} / 0.5, r_{3} / 0.4, r_{4} / 0.8\right\} \\
\} \quad & \\
\left(F_{3}, D_{3}\right)=\left\{d_{1}\right. & =\left\{r_{1} / 0.6, r_{2} / 0.7, r_{3} / 0.6, r_{4} / 0.8\right\}, \\
d_{5} & =\left\{r_{1} / 0.8, r_{2} / 0.6, r_{3} / 0.7, r_{4} / 0.4\right\}, \\
d_{6} & =\left\{r_{1} / 0.9, r_{2} / 1, r_{3} / 0.4, r_{4} / 0.6\right\}
\end{aligned}
$$

(3) The fuzzy soft sets are adopted for the information integration for $V_{1}$, $V_{2}$ and $V_{3}$, namely: implement “AND” operation for $\left(F_{1}, D_{1}\right),\left(F_{2}, D_{2}\right)$ and $\left(F_{3}, D_{3}\right)$ to $\operatorname{obtain}(G, E)=\left(G, D_{1} \times D_{2} \times D_{3}\right)=\left(F_{1}, D_{1}\right) \wedge\left(F_{2}, D_{2}\right) \wedge\left(F_{3}, D_{3}\right)$. Since $D_{1} \cap D_{2} \cap D_{3} \neq \varnothing$ is true, the number of the parameters in parameter set $E$ is $L<3 \times 3 \times 3=27$.

Firstly, we assume that $\hat{E}=\left\{\hat{e}_{1}, \hat{e}_{2}, \cdots, \hat{e}_{27}\right\}$ is obtained from the three parameters respectively provided by $D_{1}, D_{2}$ and $D_{3}$, as shown in Table 1 .

Table 1. Parameter Composition of $\hat{E}=\left\{\hat{e}_{1}, \hat{e}_{2}, \cdots, \hat{e}_{27}\right\}$

\begin{tabular}{cccccccccc}
\hline$\hat{e}_{j}$ & $\hat{e}_{1}$ & $\hat{e}_{2}$ & $\hat{e}_{3}$ & $\hat{e}_{4}$ & $\hat{e}_{5}$ & $\hat{e}_{6}$ & $\hat{e}_{7}$ & $\hat{e}_{8}$ & $\hat{e}_{9}$ \\
\hline $\begin{array}{c}\text { Original } \\
\text { Parameters }\end{array}$ & $d_{1}$ & $d_{1} d_{5}$ & $d_{1} d_{6}$ & $d_{1} d_{3}$ & $d_{1} d_{3} d_{5}$ & $d_{1} d_{3} d_{6}$ & $d_{1} d_{4}$ & $d_{1} d_{4} d_{5}$ & $d_{1} d_{4} d_{6}$ \\
\hline$\hat{e}_{j}$ & $\hat{e}_{10}$ & $\hat{e}_{11}$ & $\hat{e}_{12}$ & $\hat{e}_{13}$ & $\hat{e}_{14}$ & $\hat{e}_{15}$ & $\hat{e}_{16}$ & $\hat{e}_{17}$ & $\hat{e}_{18}$ \\
\hline $\begin{array}{c}\text { Original } \\
\text { Parameters }\end{array}$ & $d_{1} d_{2}$ & $d_{1} d_{2} d_{5}$ & $d_{1} d_{2} d_{6}$ & $d_{1} d_{2} d_{3}$ & $d_{2} d_{3} d_{5}$ & $d_{2} d_{3} d_{6}$ & $d_{1} d_{2} d_{4}$ & $d_{2} d_{4} d_{5}$ & $d_{2} d_{4} d_{6}$ \\
\hline$\hat{e}_{j}$ & $\hat{e}_{19}$ & $\hat{e}_{20}$ & $\hat{e}_{21}$ & $\hat{e}_{22}$ & $\hat{e}_{23}$ & $\hat{e}_{24}$ & $\hat{e}_{25}$ & $\hat{e}_{26}$ & $\hat{e}_{27}$ \\
\hline $\begin{array}{c}\text { Original } \\
\text { Parameters }\end{array}$ & $d_{1} d_{5}$ & $d_{1} d_{5}$ & $d_{1} d_{5} d_{6}$ & $d_{1} d_{3} d_{5}$ & $d_{3} d_{5}$ & $d_{3} d_{5} d_{6}$ & $d_{1} d_{4} d_{5}$ & $d_{4} d_{5}$ & $d_{4} d_{5} d_{6}$ \\
\hline
\end{tabular}

According to Table 2, $\hat{e}_{2}=\hat{e}_{19}=\hat{e}_{20}=\left\{d_{1} d_{5}\right\} \quad, \quad \hat{e}_{5}=\hat{e}_{22}=\left\{d_{1} d_{3} d_{5}\right\} \quad$ and $\hat{e}_{8}=\hat{e}_{25}=\left\{d_{1} d_{4} d_{5}\right\}$, so the number of the parameters in $E$ is $L=23$. If $E$ is set as $E=\left\{e_{1}, e_{2}, \cdots, e_{23}\right\}$, then the parameters of $E$ are as shown in Table 2 .

Table 2. Parameter Composition of $E=\left\{e_{1}, e_{2}, \cdots, e_{23}\right\}$

\begin{tabular}{ccccccccc}
\hline$e_{j}$ & $e_{1}$ & $e_{2}$ & $e_{3}$ & $e_{4}$ & $e_{5}$ & $e_{6}$ & $e_{7}$ & $e_{8}$ \\
\hline $\begin{array}{c}\text { Original } \\
\text { Parameters }\end{array}$ & $d_{1}$ & $d_{1} d_{5}$ & $d_{1} d_{6}$ & $d_{1} d_{3}$ & $d_{1} d_{3} d_{5}$ & $d_{1} d_{3} d_{6}$ & $d_{1} d_{4}$ & $d_{1} d_{4} d_{5}$ \\
\hline
\end{tabular}




\begin{tabular}{ccccccccc}
\hline$e_{j}$ & $e_{9}$ & $e_{10}$ & $e_{11}$ & $e_{12}$ & $e_{13}$ & $e_{14}$ & $e_{15}$ & $e_{16}$ \\
\hline $\begin{array}{c}\text { Original } \\
\text { Parameters }\end{array}$ & $d_{1} d_{4} d_{6}$ & $d_{1} d_{2}$ & $d_{1} d_{2} d_{5}$ & $d_{1} d_{2} d_{6}$ & $d_{1} d_{2} d_{3}$ & $d_{2} d_{3} d_{5}$ & $d_{2} d_{3} d_{6}$ & $d_{1} d_{2} d_{4}$ \\
\hline$e_{j}$ & $e_{17}$ & $e_{18}$ & $e_{19}$ & $e_{20}$ & $e_{21}$ & $e_{22}$ & $e_{23}$ \\
\hline $\begin{array}{c}\text { Original } \\
\text { Parameters }\end{array}$ & $d_{2} d_{4} d_{5}$ & $d_{2} d_{4} d_{6}$ & $d_{1} d_{5} d_{6}$ & $d_{3} d_{5}$ & $d_{3} d_{5} d_{6}$ & $d_{4} d_{5}$ & $d_{4} d_{5} d_{6}$ & \\
\hline
\end{tabular}

The fuzzy soft set $(G, E)$ is calculated according to formulae (8) and (9). We will take $\mu_{16}$ and $\mu_{13}$ to explain the calculation process of $(G, E)$.

$$
\begin{gathered}
\mu_{16}=\min \left\{v_{11}^{1}, v_{13}^{2}, v_{16}^{3}\right\}=\{0.9,0.9,0.9\}=0.9 \\
\mu_{13}=\min \left\{\frac{v_{11}^{1}+v_{11}^{2}}{2}, v_{16}^{3}\right\}=\min \left\{\frac{0.9+0.8}{2}, 0.9\right\}=0.85
\end{gathered}
$$

$(G, E)$ can be obtained through an analogy way, and the tabular form thereof is as shown in Table 3.

Table 3. Tabular Form of Fuzzy Soft Set $(G, E)$

\begin{tabular}{ccccccccccccc}
\hline$\mu_{t j}$ & $e_{1}$ & $e_{2}$ & $e_{3}$ & $e_{4}$ & $e_{5}$ & $e_{6}$ & $e_{7}$ & $e_{8}$ & $e_{9}$ & $e_{10}$ & $e_{11}$ & $e_{12}$ \\
\hline$r_{1}$ & 0.77 & 0.8 & 0.85 & 0.75 & 0.8 & 0.9 & 0.6 & 0.6 & 0.6 & 0.6 & 0.6 & 0.6 \\
$r_{2}$ & 0.8 & 0.6 & 0.85 & 0.75 & 0.6 & 0.8 & 0.5 & 0.5 & 0.5 & 0.7 & 0.6 & 0.7 \\
$r_{3}$ & 0.73 & 0.55 & 0.4 & 0.75 & 0.5 & 0.4 & 0.4 & 0.4 & 0.4 & 0.5 & 0.5 & 0.4 \\
$r_{4}$ & 0.73 & 0.4 & 0.6 & 0.6 & 0.4 & 0.6 & 0.75 & 0.4 & 0.6 & 0.75 & 0.4 & 0.6 \\
$\mu_{t j}$ & $e_{13}$ & $e_{14}$ & $e_{15}$ & $e_{16}$ & $e_{17}$ & $e_{18}$ & $e_{19}$ & $e_{20}$ & $e_{21}$ & $e_{22}$ & $e_{23}$ & \\
$r_{1}$ & 0.6 & 0.6 & 0.6 & 0.6 & 0.6 & 0.6 & 0.8 & 0.8 & 0.8 & 0.6 & 0.6 & \\
$r_{2}$ & 0.7 & 0.6 & 0.7 & 0.5 & 0.5 & 0.5 & 0.6 & 0.6 & 0.6 & 0.5 & 0.5 & \\
$r_{3}$ & 0.5 & 0.5 & 0.4 & 0.4 & 0.4 & 0.4 & 0.4 & 0.5 & 0.4 & 0.4 & 0.4 & \\
$r_{4}$ & 0.6 & 0.4 & 0.6 & 0.8 & 0.4 & 0.6 & 0.5 & 0.45 & 0.5 & 0.45 & 0.5 & \\
\hline
\end{tabular}

(4) The comparison table $C T=\left(c t_{x y}\right)_{4 \times 4}$ is calculated according to formulae (11) and (12), as shown in Table 4.

Table 4. Comparison Table

\begin{tabular}{ccccc}
\hline$c t_{x y}$ & $r_{1}$ & $r_{2}$ & $r_{3}$ & $r_{4}$ \\
\hline$r_{1}$ & 23 & 18 & 23 & 20 \\
$r_{2}$ & 9 & 23 & 23 & 18 \\
$r_{3}$ & 1 & 1 & 23 & 8 \\
$r_{4}$ & 8 & 6 & 17 & 23 \\
\hline
\end{tabular}

(5) The evaluation score Score $\left(r_{t}\right)$ is calculated according to formulae (13) and (14), as shown in Table 5. 
Table 5. Evaluation Score

\begin{tabular}{cccc}
\hline & $s_{x}$ & $t_{x}$ & Score $\left(r_{t}\right)$ \\
\hline$r_{1}$ & 84 & 41 & 43 \\
$r_{2}$ & 73 & 48 & 25 \\
$r_{3}$ & 33 & 86 & -53 \\
$r_{4}$ & 54 & 69 & -15 \\
\hline
\end{tabular}

According to Table 6, Score $\left(r_{1}\right)>\operatorname{Score}\left(r_{2}\right)>\operatorname{Score}\left(r_{3}\right)>\operatorname{Score}\left(r_{4}\right)$, so coalition $r_{1}$ is the best, and is orderly followed by $r_{2}, r_{3}$ and $r_{4}$.

According to the above instance, the method proposed in this article takes different individual evaluation index sets of the experts and the allowable intersection of individual evaluation index sets into consideration. The evaluations made by the experts for the coalitions are expressed by uncertain information so that the experts can flexibly express their individual subjective judgment, and the fuzzy soft set is introduced therein for the information integration of the evaluation results of the experts in order to obtain the comprehensive evaluation result.

\section{Conclusion}

In this article, the fuzzy soft set is introduced for comprehensively evaluating the coalitions. During the evaluation process, it is considered that the experts have different individual evaluation index sets and the individual evaluation index sets of the experts are allowed to be overlapped. Meanwhile, the fuzzy soft set is introduced therein to integrate the evaluation information of the experts in order to obtain the comprehensive evaluation result. In conclusion, this method can not only make comprehensive use of expert knowledge and experience, but also reflect the information uncertainty and incompleteness during the evaluation process, wherein such evaluation process is consistent with human thinking \& judgment process and has the features of flexibility, effectiveness and rationality.

\section{References}

[1] M. Zhou, G. Bao, Y. Geng, B. Alkandari and X. Li, "Polyp detection and radius measurement in small intestine using video capsule endoscopy", 2014 7th International Conference on Biomedical Engineering and Informatics (BMEI), (2014) October.

[2] G. Yan, Y. Lv, Q. Wang and Y.Geng, "Routing algorithm based on delay rate in wireless cognitive radio network", Journal of Networks, vol. 9, no. 4, (2014) January, pp. 948-955.

[3] G. Bao, L. Mi, Y.Geng and K.Pahlavan, "A computer vision based speed estimation technique for localiz ing the wireless capsule endoscope inside small intestine", 36th Annual International Conference of the IEEE Engineering in Medicine and Biology Society (EMBC), (2014) August.

[4] X. Song and Y. Geng, "Distributed community detection optimization algorithm for complex networks", Journal of Networks, vol. 9, no. 10, (2014) January, pp. 2758-2765.

[5] J. Hu and Z. Gao, "Distinction immune genes of hepatitis-induced heptatocellular carcinoma", [J]. Bioinformatics, vol. 28, no. 24, (2012), pp. 3191-3194.

[6] J. Hu, Z. Gao and W. Pan, "Multiangle Social Network Recommendation Algorithms and Similarity Network Evaluation", Journal of Applied Mathematics, (2013).

[7] J. Hu and Z. Gao, "Modules identification in gene positive networks of hepatocellular carcinoma using Pearson agglomerative method and Pearson cohesion coupling modularity", Journal of Applied Mathematics, (2012).

[8] X. Zhang, "Spike-based indirect training of a spiking neural network-controlled virtual insect", 2013 IEEE 52nd Annual Conference on Decision and Control (CDC), IEEE, (2013).

[9] K. Wang, "Towards Scalable Distributed Workload Manager with Monitoring-Based Weakly Consistent Resource Stealing", (2015). 
[10] Z. Lv, T. Yin, Y. Han, Y. Chen and G. Chen, "WebVR-web virtual reality engine based on P2P network", Journal of Networks, vol. 6, no. 7, (2011), pp. 990-998.

[11] J. Yang, B. Chen, J. Zhou and Z. Lv, "A portable biomedical device for respiratory monitoring with a stable power source", Sensors, (2015).

[12] S.Dang, J. Ju, D. Matthews, X. Feng and C. Zuo, "Efficient solar power heating system based on lenticular condensation. Information Science", Electronics and Electrical Engineering (ISEEE), 2014 International Conference, (2014) April 26-28.

[13] X. Zhang, Y. Han, D. Hao and Z. Lv, “ARPPS : Augmented Reality Pipeline Prospect System”, 22th International Conference on Neural Information Processing (ICONIP), Istanbul, Turkey. In press, (2015).

[14] K. Wang, "Overcoming Hadoop Scaling Limitations through Distributed Task Execution".

[15] Su Zhang, X. Zhang, and X. Ou, "After we knew it: empirical study and modeling of cost-effectiveness of exploiting prevalent known vulnerabilities across iaas cloud", Proceedings of the 9th ACM symposium on Information, computer and communications security, ACM, (2014).

[16] W. Gu, Z. Lv and M. Hao, "Change detection method for remote sensing images based on an improved Markov random field", Multimedia Tools and Applications, (2016).

[17] Z. Lu, C. Esteve, J. Chirivella and P. Gagliardo, "A Game Based Assistive Tool for Rehabilitation of Dysphonic Patients", 3rd International Workshop on Virtual and Augmented Assistive Technology (VAAT) at IEEE Virtual Reality 2015 (VR2015), Arles, France, IEEE, (2015).

[18] Z. Chen, W. Huang and Z. Lv, "Uncorrelated Discriminant Sparse Preserving Projection Based Face Recognition Method", Multimedia Tools and Applications, (2016).

[19] Z. Lv, A.Halawani, S. Feng, H. Li, and S. Ur Rehman, "Multimodal Hand and Foot Gesture Interaction for Handheld Devices. ACM Transactions on Multimedia Computing, Communications, and Applications", (TOMM), vol. 11, no. 1, (2014) October 10, pp. 19.

[20] K. Leng, W. Shi, J. Chen and Z. Lv, "Designing of a I-shaped less-than-truckload cross-dock: A simulation experiments study", International Journal of Bifurcation and Chaos, (2015).

[21] Y. Lin, J. Yang, Z. Lv, W. Wei and H. Song, "A Self-Assessment Stereo Capture Model Applicable to the Internet of Things", Sensors, (2015).

[22] W. Ou, Zhihan Lv and Zanfu Xie, "Spatially Regularized Latent topic Model for Simultaneous object discovery and segmentation", The 2015 IEEE International Conference on Systems, Man, and Cybernetics (SMC), (2015).

[23] K. Wang, "Using Simulation to Explore Distributed Key-Value Stores for Exascale System Services", 2nd Greater Chicago Area System Research Workshop (GCASR), (2013).

[24] Y. Wang, Y. Su and G. Agrawal, "A Novel Approach for Approximate Aggregations Over Arrays", Proceedings of the 27th international conference on scientific and statistical database management, ACM, (2015).

[25] Z. Lv, A. Halawani, S. Feng, S. ur Rehman and H. Li, "Touch-less Interactive Augmented Reality Game on Vision Based Wearable Device", Personal and Ubiquitous Computing, (2015).

[26] J. Yang, S. He, Y. Lin and Z. Lv, "Multimedia cloud transmission and storage system based on internet of things", Multimedia Tools and Applications, (2016).

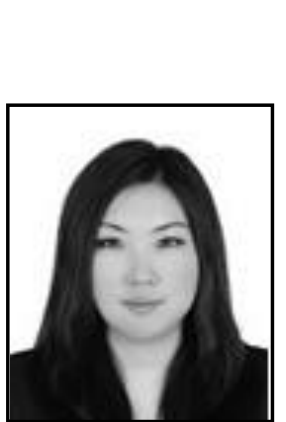

\section{Author}

Jun Qi was born in HeiBei, China, in 1982. She received her Msc and Phd degree in Computational Finance from Essex University at UK, in 2005 and 2012, respectively. She is currently a lecturer in the Jinan University at Guangzhou, China. Her research interest is mainly in the area of Computational Finance, Corporate Finance and Big data Finance. She has published several research papers in scholarly journals in the above research areas. 
International Journal of $u-$ and e- Service, Science and Technology Vol.9, No. 4 (2016) 